Article

\title{
On the Inception of Financial Representative Bubbles
}

\author{
Massimiliano Ferrara $1,2,3, *,+$ (D), Bruno A. Pansera $1,2,+$ and Francesco Strati $1,2,+$ \\ 1 Department of Law and Economics, University Mediterranea of Reggio Calabria, Via dei Bianchi 2, \\ 89127 Reggio Calabria, Italy; bruno.pansera@unirc.it (B.A.P.); francesco.strati@unirc.it (F.S.) \\ 2 Decisions Lab, Department of Law and Economics, University Mediterranea of Reggio Calabria, \\ Via dei Bianchi 2, 89127 Reggio Calabria, Italy \\ 3 Invernizzi Center for Research on Innovation, Organization, Strategy and Entrepreneurship, \\ Department of Management and Technology, Bocconi University, Via Sarfatti 25, 20136 Milano, Italy \\ * Correspondence: massimiliano.ferrara@unirc.it \\ + These authors contributed equally to this work.
}

Received: 9 October 2017; Accepted: 14 November 2017; Published: 17 November 2017

\begin{abstract}
In this work, we aim to formalize the inception of representative bubbles giving the condition under which they may arise. We will find that representative bubbles may start at any time, depending on the definition of a behavioral component. This result is at odds with the theory of classic rational bubbles, which are those models that rely on the fulfillment of the transversality condition by which a bubble in a financial asset can arise just at its first trade. This means that a classic rational bubble (differently from our model) cannot follow a cycle since if a bubble exists, it will burst by definition and never arise again.
\end{abstract}

Keywords: financial mathematics; financial bubbles; behavioral finance

\section{Introduction}

In Diba and Grossman [1] (following the results of Tirole [2]), it has been shown that a rational bubble rises only if it is already present at the first trading of a bubbly asset, that is: if a bubble does not blow at the first trade, it cannot rise at any subsequent time. Moreover, Giglio et al. [3] tested for the existence of rational bubbles in the housing market that preceded the turmoil of 2007: they found that there has not been a failure of the transversality condition, and then, rational bubbles can be ruled out as a culprit for overvalued houses. Furthermore, Strati [4] defined a behavioral model of the bubble that is able to consider over-optimism (respectively over-pessimism) in the market by taking into account a behavioral component that is neglected in the classic rational case, which can detect a growing bubble even in cases in which the transversality condition is fulfilled (that is, when the intertemporal equilibrium of prices is fulfilled). A key component of Strati [4] is that a bubble may arise because of an exogenous flow of positive news that overheats the perception of a so-called local thinker who is excessively sensitive to new information. In this paper, we aim to clarify the formal inception of this kind of behavioral bubble driven by the heuristic of representativeness (Kahneman and Tversky [5], Kahneman and Tversky [6], Tversky and Kahneman [7], Tversky and Kahneman [8]): the representative bubble (in financial economics, the application of non-Gaussian processes as the Ornstein-Uhlenbec, aiming at similar questions; see for example Issaka and SenGupta [9]). We will find that a representative bubble may blow at any time; hence, it is at odds with the inception of a rational bubble as defined in Diba and Grossman [1]. In particular, the model takes into account homogeneous expectations and a shared euphoria among agents caused by a flow of positive news. These positive shocks, or displacements (Kindleberger [10]), inflate the probability of an expected growth state: it is a diagnostic signal of their representativeness; thus, the expectations that follow from this heuristic are called diagnostic. The representative expected state inflates with respect to the rational expectations 
component, that is to say, diagnostic expectations exaggerate the direction of the path followed by the rational ones without changing the direction: diagnostic expectations are based upon a kernel of truth. These behavioral expectations are not adaptive in nature; they are forward-looking and thus immune to the Lucas critique. The heuristic of this kind of expectations has been examined by Kahneman and Tversky [5,6], and recently formalized in Gennaioli and Shleifer [11]. However, the representativeness heuristic can be defined in several ways (see Bordalo et al. [12]); here, we follow the so-called representativeness-based discounting used in Bordalo et al. [13] and Strati [4] (see also [14] for an application to financial fragility in which the representativeness heuristic completely neglects downside risks).

\section{The Inception and the Representativeness}

In this section, we shall define the condition by which representative bubbles can inflate at any time: their inception is linked to the level of rationality of the agents. From Diba and Grossman [1], it will be clear that for fully-rational agents, if a rational bubble does not exist at $t \geq 0$, then that rational bubble can get started neither at $t+1$, nor at any subsequent time (Diba and Grossman [1]). However, this result of Diba and Grossman [1] is not true when agents are local thinkers, that is those agents whose expectations are driven by the heuristic of representativeness.

\subsection{The Rational Case}

Following Diba and Grossman [1], we define a first order stochastic difference Equation (1) in $y$ with a forcing variable $x$ and a set of information $I_{t}$, a set containing past realizations of both exogenous and endogenous variables up to current time $t$. We describe a rational asset-price bubble in the context of (linear) rational expectations $\mathbb{E}_{t}[\cdot]$ (the rational expectations of a variable of a model formed by an individual at time $t$ are defined as the mathematical expectation conditional on the information set $I_{t}$ ), that is:

$$
\alpha \mathbb{E}_{t}\left[y_{t+1} \mid I_{t}\right]=y_{t}-x_{t}
$$

For the eigenvalue $\alpha>1$, Equation (1) is convergent; it can be solved by the forward-looking method (note: $\alpha=\frac{1}{1+r}$ with $r>0$, which can be thought of as an interest rate). In particular, by applying the law of iterated expectations, that is to say, by leading and taking expectations of (1):

$$
\mathbb{E}\left[y_{t+1} \mid I_{t}\right]=\alpha \mathbb{E}\left[\mathbb{E}\left[y_{t+2} \mid I_{t}\right]\right]+\mathbb{E}\left[x_{t+1} \mid I_{t}\right]
$$

we obtain:

$$
\begin{aligned}
y_{t} & =\alpha \mathbb{E}_{t}\left[y_{t+1} \mid I_{t}\right]+x_{t} \\
& =\alpha^{2} \mathbb{E}_{t}\left[y_{t+2} \mid I_{t}\right]+\alpha \mathbb{E}_{t}\left[x_{t+1} \mid I_{t}\right]+x_{t} \\
& \cdots \\
& =\alpha^{T+1} \mathbb{E}_{t}\left[y_{t+1+T} \mid I_{t}\right]+\sum_{i=0}^{T} \alpha^{i} \mathbb{E}_{t}\left[x_{t+i} \mid I_{t}\right],
\end{aligned}
$$

taking into account that if $T=0$, then the expectation at $T=0$ coincides with $x_{t}$ and $I_{t}=I$ for every $t \in\{0, \ldots, T\}$. The forward-looking solution, or fundamental solution, is the second term of the right-hand side of (2), and it is usually denoted by $F_{t}$, that is:

$$
F_{t}=\sum_{i=0}^{T} \alpha^{i} \mathbb{E}_{t}\left[x_{t+i} \mid I_{t}\right] .
$$

The first term of the right-hand side of (2) is called the bubble component $B_{t}$ and from which is obtained the bubble solution. In order to have a unique solution of (1), the transversality condition on the bubble component should be imposed: 


$$
\lim _{T \rightarrow \infty} \alpha^{T+1} \mathbb{E}_{t}\left[y_{t+1+T} \mid I_{t}\right]=0,
$$

otherwise $y_{t}=F_{t}$, which would have been only one of the possible solutions of:

$$
y_{t}=F_{t}+B_{t} .
$$

In this general case, $B_{t}$ is the solution of the stochastic difference equation:

$$
\alpha \mathbb{E}_{t}\left[B_{t+1} \mid I_{t}\right]-B_{t}=0
$$

for which:

$$
B_{t+1}-\alpha^{-1} B_{t}=z_{t+1} .
$$

Note that $\mathbb{E}_{t-j} z_{t+1}=0$ for all $j \geq 0$. From the latter property, Diba and Grossman [1] found a convincing result: a bubble cannot blow at $t+i$ if it is not already present at $t$, at the inception. From Diba and Grossman [1], the bubble component can be seen as an innovation comprising new information available at $t+1$. The information encompassed in those changes of $x_{t}$ can affect $y_{t}$ directly or by modifying the information set $I_{t}=\left\{x_{t}, x_{t-1}, \ldots\right\}$, hence affecting $y_{t}$ indirectly by the expectation term in (1). The bubble component may not be connected with the fundamental information, and since the expected future values of $z_{t+i}$ are zero by definition of the rational model, then the new information of innovation seems not to be of any help in the assessment of a positive $B_{t+1}$.

From Equation (5), it follows that $\mathbb{E}_{t}\left[B_{t+j} \mid I_{t}\right]=\alpha^{-j} B_{t}$; there is an explosive conditional expectation on the value of the bubble component for $B_{t}>0$, and $\mathbb{E}_{t}\left[B_{t+j}\right]=\infty$ for $j \rightarrow \infty$ and $1 / \alpha>1$. However, if $y_{t}$ is a (asset) price, then by the free disposal of the Walrasian equilibrium, $y_{t} \geq 0$, the possibility of a negative rational bubble is ruled out. If the free disposal implies a nonnegativity for $y_{t}$, then the bubble component of $y_{t+1}$ must satisfy $B_{t+1} \geq 0$. From Equation (6) and the nonnegativity condition:

$$
z_{t+1} \geq-\lambda B_{t}
$$

Assuming that at $t, B_{t}=0$, since $\mathbb{E}_{t-j} z_{t+1}=0$, then in this case, the probability of a $z_{t+1}=0$ is equal to one. By Equation (6), the bubble component is equal to the innovation brought about by $z_{t+1}$; hence, if one is zero, the other must be zero: if a bubble exists, it must exist from the inception. Another property follows from the transversality condition of Equation (4), that is: if there is a departure from the optimal path of $y_{t+i}$ and thus a failure of the transversality condition (4), then from the rational expectations equilibrium, arbitrageurs adjust the inflated value of $y_{t+i}$ at once, using up the positive bubble component.

\subsection{A Brief Digression: The Representativeness}

In this section, we shall review the meaning of the heuristic that causes errors in the formation of the agents's expectations, the representativeness heuristic. These biased expectations will be defined as diagnostic expectations.

In Kahneman and Tversky [6], the representativeness is defined as "an assessment of the degree of the correspondence between a sample and a population..., more generally, between an outcome and a model". In other words: "A person who follows this heuristic evaluates the probability of an uncertain event, or a sample, by the degree to which it is: (i) similar in essential properties to its parent population; and (ii) reflects the salient features of the process by which it is generated" (Kahneman and Tversky [5]). For a distribution of a trait $T$ in a group $\Gamma$, a decision maker (DM) will assess the relative frequency of a particular trait $T=t$ in $\Gamma$ with respect to the frequency of the same trait $T=t$ in a relevant comparison group $\Gamma^{c}$. The true distribution is $\operatorname{Pr}(T=t \mid \Gamma)$. The heuristic of representativeness $\mathcal{R}(\cdot)$ has been formalized in Gennaioli and Shleifer [11] and Bordalo et al. [12]; in particular, from Gennaioli and Shleifer [11], the representativeness can be modeled as follows: 


$$
\frac{\operatorname{Pr}(T=t \mid \Gamma)}{\operatorname{Pr}\left(T=t \mid \Gamma^{c}\right)}
$$

It is plain that representativeness defines the inflation of the likelihood of traits whose objective probability rises the most in $\Gamma$ relative to the reference context $\Gamma^{c}$ (Bordalo et al. [13]). Let us show how this formalization modifies expectations.

Diagnostic Expectations

There is a discrete time $t=1,2, \ldots$ and two states of the world: growth $g$ and recession $r$. At any time, there is a signal $s \in \mathbb{R}_{+}$about the next state space, with a growth signal $\bar{s}$ and a recession signal $\underline{s}$ with $\underline{s}<\bar{s}$. The signals are characterized in the following way: $\operatorname{Pr}(\underline{s} \mid r)=\gamma, \operatorname{Pr}(\underline{s} \mid g)=1-\beta$ for which $\gamma>\beta \geq 1 / 2$ : a bad signal $\underline{s}$ reduces the probability of expecting a growth rate, and it is a very strong signal for a looming recession.

Assumption 1. There is a prior probability $\pi_{k}$ with $k=\{g, r\}$ for which $\pi_{g}>\pi_{r}$.

Denote a generic state space $\Omega$ in which $\omega$ is a realization. In particular, the state of the economy is a random variable $\Omega_{t}$. Define by $\left(\Omega_{t+1}=\omega_{t+1}\right)$ the realization of the state $\omega_{t+1}$ at $t+1$. If $\left(\Omega_{t+1}=\omega_{t+1} \mid \Omega_{t}=\omega_{t}\right)$ then it is intended that the realization $\omega_{t+1}$ at $t+1$ hinges on the occurrence of the state $\omega_{t}$ at $t$. Now, suppose that there exists a smooth density function $f(\cdot)$ for which in a Bayesian framework:

$$
F\left(s \mid \omega_{t+1}\right)=\int_{s}^{+\infty} f\left(\Omega_{t+1}=\omega_{t+1} \mid \Omega_{t}=\omega_{t}\right) d \omega_{t+1}
$$

is the rational cumulative distribution of the probability that the state $\omega_{t+1}$ is a growth state.

Define $\Gamma \equiv\left\{\Omega_{t}=\omega_{t}\right\}$ as the group that depicts all the possible future states $\omega_{t+1}$ whose values depend on the current state $\Omega_{t}=\omega_{t}$. Moreover, define its comparison group $\Gamma^{c} \equiv \Omega \backslash \Gamma$. As in Bordalo et al. [13], the comparison group may be defined as the rational expectation formed at $t-1$ for $\omega_{t+1}$ in which no new pieces of information occur, that is $\Gamma^{c} \equiv\left\{\Omega_{t}=\mathbb{E}_{t-1}\left(\omega_{t}\right)\right\}$ (it is the background context, the state prevailing if there is no news).

Definition 1. The representativeness of a state $\omega_{t}$ at $t$ for a group $\Gamma$ is defined as:

$$
\mathcal{R}\left(\omega_{t}, \Gamma, \Gamma^{c}\right)=\frac{f\left(\Omega_{t+1}=\omega_{t+1} \mid \Omega_{t}=\omega_{t}\right)}{f\left(\Omega_{t+1}=\omega_{t+1} \mid \Omega_{t}=\mathbb{E}_{t-1}\left(\omega_{t}\right)\right)} .
$$

In particular, following Bordalo et al. [13] and Strati [4], the representativeness is set up by considering a mental process that formalizes the similarity between group $\Gamma \equiv\left\{\Omega_{t}=\omega_{t}\right\}$ and the comparison group, by considering a limited and selective retrieval from memory: how scenarios become accessible from memory. They come to mind following an order dictated by their representativeness rather than by their probability. This mental process is formalized as follows:

$$
d\left(\omega_{t+1}\right) \equiv f\left(\Omega_{t+1}=\omega_{t+1} \mid \Omega_{t}=\omega_{t}\right) \times\left[\frac{f\left(\Omega_{t+1}=\omega_{t+1} \mid \Omega_{t}=\omega_{t}\right)}{f\left(\Omega_{t+1}=\omega_{t+1} \mid \Omega_{t}=\mathbb{E}_{t-1}\left(\omega_{t}\right)\right)}\right]^{\theta} \frac{1}{Z}
$$

note that $1 / Z$ is a normalizing constant that assures the integrability to be one of (10). In short, from Equation (10), outcomes are ordered by the degree to which they are representative of specific evidence; the statistical theory of prediction is not fully considered. The model detects this confusion between likelihood and representativeness that leads agents to erroneously predict future events. 


\subsection{The Representative Case}

In this section, we enrich the model of rational bubbles by considering diagnostic expectations. From the reasoning on the inception of rational bubbles, some conclusions can be stated: a rational bubble is present from the inception of a trade, and when it bursts, it cannot arise again in the same asset. Moreover, a bubble component can be positive just for a very short span of time. However, from the bubble defined in Strati [4], these conclusions should be revised.

Define Equation (1) in the following way:

$$
y_{t}=\alpha \mathbb{E}_{t}^{d}\left[y_{t+1}\right]+x_{t}
$$

in which $\mathbb{E}_{t}^{d}[\cdot]$ is the diagnostic expectation from which the representative density function (10) is explicitly defined as a normal distribution:

$$
d\left(y_{t+1}\right)=\frac{1}{\sigma \sqrt{2 \pi}} \exp \left(-\frac{\left(y_{t+1}-\mathbb{E}_{t}\left(y_{t+1}\right)\right)^{2}}{2 \sigma^{2}}\right) \times \frac{\left[\exp \left(-\frac{\left(y_{t+1}-\mathbb{E}_{t}\left(y_{t+1}\right)\right)^{2}}{2 \sigma^{2}}\right)\right]^{\theta}}{\left[\exp \left(-\frac{\left(y_{t+1}-\mathbb{E}_{t-1}\left(y_{t+1}\right)\right)^{2}}{2 \sigma^{2}}\right)\right]^{\theta}} \frac{1}{Z}
$$

such that, by exploiting the exponential property:

$$
e^{-a}\left(\frac{e^{-a}}{e^{-b}}\right)^{\theta} \Rightarrow e^{\theta b-(\theta+1) a} \text { for some } a, b \in \mathbb{R}
$$

we obtain:

$$
d\left(y_{t+1}\right)=\frac{1}{\sigma \sqrt{2 \pi}} \exp \left(-\frac{1}{2 \sigma^{2}}\left[(1+\theta)\left(y_{t+1}-\mathbb{E}_{t}\left(y_{t+1}\right)\right)^{2}-\theta\left(y_{t+1}-\mathbb{E}_{t-1}\left(y_{t+1}\right)\right]^{2}\right) \frac{1}{Z}\right.
$$

Moreover, since:

$$
\begin{aligned}
{\left[(1+\theta)\left(y_{t+1}-\mathbb{E}_{t}\left(y_{t+1}\right)\right)^{2}-\theta\left(y_{t+1}-\mathbb{E}_{t-1}\left(y_{t+1}\right)\right]^{2}\right.} & =\left(y_{t+1}-\mathbb{E}_{t}\left(y_{t+1}\right)+\theta\left[\mathbb{E}_{t}\left(y_{t+1}\right)-\mathbb{E}_{t-1}\left(y_{t+1}\right)\right]\right)^{2} \\
& =\left(y_{t+1}-\mathbb{E}_{t}^{d}\left(y_{t+1}\right)\right)^{2}
\end{aligned}
$$

we rewrite Equation (12) as:

$$
d\left(y_{t+1}\right)=\frac{1}{\sigma \sqrt{2 \pi}} \exp \left(-\frac{1}{2 \sigma^{2}}\left[\left(y_{t+1}-\mathbb{E}_{t}^{d}\left(y_{t+1}\right)\right)^{2}\right]\right) \frac{1}{Z} .
$$

In this linear environment, it is plain how the heuristic of representativeness causes an exaggeration in the judgment based on rational expectations, that is:

$$
\mathbb{E}_{t}^{d}\left(y_{t+1}\right) \equiv \mathbb{E}_{t}\left(y_{t+1}\right)+\theta\left[\mathbb{E}_{t}\left(y_{t+1}\right)-\mathbb{E}_{t-1}\left(y_{t+1}\right)\right]
$$

in which $\theta$ shifts the (Gaussian) rational distribution to the right. Since the rational (Gaussian) distribution remains the feasible one for the market, then this shift causes a neglection of the tail risk encompassed in the (Gaussian) rational distribution and over-optimism: good news causes neglect of downside risks and an increasing optimism. In Strati [4], it has been shown that for positive shocks to fundamentals, the so-called displacements (see Kindleberger [10]), there is an excess of volatility that brings about a positive forecast error that, since it is not orthogonal to the information available at the time of the forecast, triggers the presence of a positive component directly into the fundamental solution. This result tells us that there is no need for a positive bubble component: a representative bubble (driven by representativeness) overvaluation anyway. Let us see this reasoning formally. 


\subsubsection{Diagnostic Expectations: Extrapolation and Neglect}

The excess volatility is justified by the fact that rational expectations are distorted towards the direction of realized news (see Greenwood and Shleifer [15] for empirical results). Positive news can cause over-optimistic expectations (vice versa for negative news). Consider the cumulative distribution:

$$
F_{t}^{d}(s)=\int_{s}^{+\infty} d\left(y_{t+1}\right) d y_{t+1},
$$

that describes the probability that positive news continues to hit the model. Note that for a contingent current value $y_{t}$ :

$$
\frac{\partial \ln F_{t}^{d}(s)}{\partial y_{t}}=\frac{1}{F_{t}^{d}(s)} \frac{\partial F_{t}^{d}(s)}{\partial \mathbb{E}_{t}^{d}\left(y_{t+1}\right)} \cdot \frac{\partial \mathbb{E}_{t}^{d}\left(y_{t+1}\right)}{\partial y_{t}} .
$$

This is important because one can show that positive news is extrapolated to the next future date. We need to follow two steps:

(i) the first term on the right-hand side is:

$$
\begin{aligned}
\frac{1}{F_{t}^{d}(s)} \frac{\partial F_{t}^{d}(s)}{\partial \mathbb{E}_{t}^{d}\left(y_{t+1}\right)} & =\int_{\bar{s}}^{\infty} \frac{y_{t+1}-\mathbb{E}_{t}^{d}\left(y_{t+1}\right)}{\sigma^{2}} \cdot \exp \left[-\frac{\left(y_{t+1}-\mathbb{E}_{t}^{d}\left(y_{t+1}\right)\right)^{2}}{2 \sigma^{2}}\right] \frac{d y_{t+1}}{F_{t}^{d}(s)} \\
& =\frac{1}{\sigma^{2}} \int_{\bar{s}}^{\infty} y_{t+1} \exp \left(-\frac{1}{2 \sigma^{2}}\left[\left(y_{t+1}-\mathbb{E}_{t}^{d}\left(y_{t+1}\right)\right)^{2}\right]\right) \frac{d y_{t+1}}{\partial F_{t}^{d}(s)}-\frac{\mathbb{E}_{t}^{d}\left(y_{t+1}\right)}{\sigma^{2}}
\end{aligned}
$$

that is to say:

$$
\frac{\partial \ln F_{t}^{d}(s)}{\partial y_{t}}=\left[\mathbb{E}_{t}^{d}\left(y_{t+1} \mid y_{t+1} \geq \bar{s}\right)-\mathbb{E}_{t}^{d}\left(y_{t+1}\right)\right] \frac{1}{\sigma^{2}} ;
$$

(ii) as for the second term, assume normal densities and an autoregressive model of order one, $\operatorname{AR}(1)$ for $\mathbb{E}_{t}\left(y_{t+1}\right)=a+b y_{t}$, rewriting (13) in these terms, then:

$$
\frac{\partial \mathbb{E}_{t}^{d}\left(y_{t+1}\right)}{\partial y_{t}}=b(1+\theta)>0 .
$$

It follows that:

$$
\frac{\partial \ln F_{t}^{d}(s)}{\partial y_{t}}>0 .
$$

From Equations (15) and (16), an over-optimistic behavior is driven by $\theta$ (the extent of the representativeness) that triggers an excess of volatility detected by the variance:

$$
\begin{aligned}
\operatorname{Var}\left[\mathbb{E}_{t}^{d}\left(y_{t+1}\right)\right] & =\operatorname{Var}\left[(1+\theta) \mathbb{E}_{t}\left(y_{t+1}\right)-\mathbb{E}_{t-1}\left(y_{t+1}\right)\right] \\
& =(1+\theta)^{2} \operatorname{Var}\left[\mathbb{E}_{t}\left(y_{t+1}\right)\right] .
\end{aligned}
$$

that in turn, taking into account Equation (13), sets off a positive predictable error:

$$
\begin{aligned}
\mathbb{E}_{t}\left[y_{t+1}-\mathbb{E}_{t}^{d}\left(y_{t+1}\right)\right] & =\mathbb{E}_{t}\left(y_{t+1}-\left(\mathbb{E}_{t}\left(y_{t+1}\right)+\theta\left[\mathbb{E}_{t}\left(y_{t+1}\right)-\mathbb{E}_{t-1}\left(y_{t+1}\right)\right]\right)\right) \\
& =\mathbb{E}_{t}\left(y_{t+1}\right)-\mathbb{E}_{t}\left(\mathbb{E}_{t}\left(y_{t+1}\right)\right)+\mathbb{E}_{t}\left(\theta \mathbb{E}_{t}\left(y_{t+1}\right)\right)-\mathbb{E}_{t}\left(\theta\left(\mathbb{E}_{t-1}\left(y_{t+1}\right)\right)\right. \\
& =-\theta\left[\mathbb{E}_{t}\left(y_{t+1}\right)-\mathbb{E}_{t-1}\left(y_{t+1}\right)\right] .
\end{aligned}
$$

Since past values of $y$ affect the formation of the current expectations, then the orthogonality condition is violated due to the extrapolation of past good news. Moreover, the negative term of the 
error is justified by the fact that an ex-ante overvaluation is symptomatic of a future fall of the value (see for example Baron and Xiong [16] or Gennaiolo et al. [17]).

\subsubsection{The Representative Bubble}

As for the rational case, let us find a forward solution (now) for Equation (11), that is:

$$
y_{t}=\alpha^{T+1} \mathbb{E}_{t-1+T}^{d}\left[y_{t+1+T} \mid I_{t}\right]+\sum_{i=0}^{T} \alpha^{i} \mathbb{E}_{t+1}^{d}\left[x_{t+i} \mid I_{t}\right] .
$$

Since we are interested in the case in which a bubble exists also in the case of a zero bubble component, consider that the transversality condition is fulfilled, and thus, even for a $\theta>0$, Equation (13) tends to zero due to the free disposal on $t-1$.

Assumption 2. $\lim _{T \rightarrow \infty} \mathbb{E}_{t}\left[\alpha_{t, t+T-1} p_{t+T-1}\right]=0$,

From Assumption 2, it follows that:

$$
y_{t}=\sum_{i=0}^{T} \alpha^{i} \mathbb{E}_{t+1}^{d}\left[x_{t+i} \mid I_{t}\right]
$$

Differently from Equation (3), in Equation (19), expectations are assumed to be diagnostic. In particular, agents are over-sensitive to positive news that hits fundamentals (displacements). This biased sensitivity causes an overconfidence among agents that in turn triggers both a neglection of downside risks and a positive predictable error due to the extrapolative nature of these expectations. By implicitly assuming that the feasible path is that which follows rational expectations, the difference between the path driven by representativeness and that of the rational one suggests that for Equation (19), the reasoning behind the forward solution has to be different. It is now clear that the fundamental value of Equation (19) is biased by a positive error. This error, as we stated, derives from the excess of volatility of (17); that is:

$$
\epsilon_{t}^{d}=\left[\mathbb{E}_{t}^{d}\left(x_{t+1}\right)-\mathbb{E}_{t}\left(x_{t+1}\right)\right]
$$

that, from Equation (13), can be rewritten as:

$$
\epsilon_{t}^{d} \equiv \theta\left[\mathbb{E}_{t}\left(x_{t+1}\right)-\mathbb{E}_{t-1}\left(x_{t+1}\right)\right]
$$

where $\epsilon^{d}$ is the error in expectations caused by diagnostic expectations. The solution of Equation (19) is thus:

$$
y_{t}=\sum_{i=0}^{T} \alpha^{i} \mathbb{E}_{t+1}\left[x_{t+i} \mid I_{t}\right]+\epsilon_{t}^{d}
$$

with $\epsilon_{t}^{d}>0$, that is $y_{t}=F_{t}+\epsilon_{t}^{d}$. In Strati [4], it has been shown that for a homogeneous euphoria of the market, which can be not detected by rational bubbles (due to the transversality condition on $B_{t}$ ), there can exist a bubble driven by representativeness.

\subsubsection{When Does It Start?}

Differently from the rational case, if a bubble is representative, that bubble can start (respectively burst) at any time, depending on both the persistence and sensitivity of the agents's expectations to positive (respectively negative) shocks. The level of rationality of the agents is thus crucial for the inception of a bubble that does not always coincide with the first trade of an asset. Moreover, the 
parameter $\theta$ can either increase or decrease with respect to the institutions in which agents operate, or with respect to how and what comes to mind when they make a decision.

Proposition 1. A bubble driven by representativeness can arise in the related environment at any $t \geq 0$.

Proof. By Assumption 2, it is plain that the bubble component is zero even if $\theta>0$. The fundamental solution brings about a positive expected error $\epsilon^{d}$ because of the excess volatility and the subsequent positive predictable error. Since it is assumed that a positive shock hits the model, by Equation (16), agents overreact to positive shocks to fundamentals causing the strict positivity of $\epsilon^{d}$. Since $\theta>0$, Equation (21) is fulfilled: $y_{t}>F_{t}$ by the strictly positive error in expectations.

Remark 1. The representative bubble is caused by a limited rationality of the agents for every credible shock that hits agents's beliefs and that can occur at any $t \geq 0$.

\section{Conclusions}

In this work, we showed that representative bubbles may blow at any time and with no restrictions. The implications are thus the followings:

- From Proposition 1, a representative bubble can start at any time due to displacements that follow from extrapolative expectations and neglect of tail risks. In short, from diagnostic expectations;

- As rational bubbles, the representative dynamics cannot be negative due to the free disposal;

- Following Proposition 1, representative bubbles can arise, burst at zero and then grow again in the same asset;

- A representative bubble is not dependent on the failure of the transversality condition; hence, it can be persistent and distorted.

This work considers just homogeneous expectations among agents. An improvement should be made towards models provided with heterogeneous expectations so as to study how rational agents can react to the presence of local thinkers.

Author Contributions: All authors contributed to the technical analysis and development of the results.

Conflicts of Interest: The authors declare no conflict of interest.

\section{References}

1. Diba, T.B.; Grossman, I.H. On the Inception of Rational Bubbles. Q. J. Econ. 1987, 102, 697-700.

2. Tirole, J. On the Possibility of Speculation under Rational Expectations. Econometrica 1982, 50, 1163-1181.

3. Giglio, S.; Maggiori, M.; Stroebel, J. No-Bubble Condition: Model-Free Tests in Housing Markets. Econometrica 2016, 84, 1047-1091.

4. Strati, F. Three Essays on Behavioral Economics. Ph.D. Thesis, University of Siena, Siena, Italy, 2017.

5. Kahneman, D.; Tversky, A. Subjective Probability: A Judgment of Representativeness. Cogn. Psychol. 1972, 3, 430-454.

6. Kahneman, D.; Tversky, A. Extensional versus Intuitive Reasoning: The Conjunction Fallacy in Probability Judgment. Psychol. Rev. 1983, 90, 293-315.

7. Tversky, A.; Kahneman, D. Judgment under Uncertainty: Heuristics and Biases. Science 1974, 185, $1124-1131$.

8. Tversky, A.; Kahneman, D. Belief in the Law of Small Numbers. Psychol. Bull. 1971, 76, 105-110.

9. Issaka, A.; SenGupta, I. Analysis of variance based instruments for Ornstein-Uhlenbeck type models: Swap and price index. Ann. Financ. 2017, 13, 401-434.

10. Kindleberger, C. Manias, Panics, and Crashes: A History of Financial Crises; Basic Books: New York, NY, USA, 1978.

11. Gennaioli, N.; Shleifer, A. What Comes to Mind. Q. J. Econ. 2010, 125, 1399-1433.

12. Bordalo, P.; Coffman, K.; Gennaioli, N.; Shleifer, A. Stereotypes. Q. J. Econ. 2016, 131, 1753-1794.

13. Bordalo, P.; Gennaioli, N.; Shleifer, A. Diagnostic Expectations and Credit Cycles. J. Financ. 2017, in press. 
14. Gennaioli, N.; Shleifer, A.; Vishny, R. Neglected Risks, Financial Innovation, and Financial Fragility. J. Financ. Econ. 2012, 104, 452-468.

15. Greenwood, R.; Shleifer, A. Expectations of Returns and Expected Returns. Rev. Financ. Stud. 2014, 27, 714-746.

16. Baron, M.; Xiong, W. Credit Expansion and Neglected Crash Risk. Q. J. Econ. 2017, 132, 713-764.

17. Gennaioli, N.; Ma, Y.; Shleifer, A. Expectations and Investment. NBER Macroecon. Annu. 2015, 30, 379-442.

(C) 2017 by the authors. Licensee MDPI, Basel, Switzerland. This article is an open access article distributed under the terms and conditions of the Creative Commons Attribution (CC BY) license (http:/ / creativecommons.org/licenses/by/4.0/). 